\title{
Conservation status of plant resources of Hazar Nao hills, district Malakand, Pakistan
}

Zahir Muhammad ${ }^{1}$, Hazrat Ali ${ }^{1}$, Wisal Muhammad Khan ${ }^{2 *}$, Rehmanullah ${ }^{1}$, Ghulam Jelani ${ }^{1}$ and Abdul Majeed ${ }^{3}$

1. Department of Botany, University of Peshawar-Pakistan

2. Department of Botany, Islamia College, Peshawar-Pakistan

3. Department of Botany, Government Degree College Naguman Peshawar-Pakistan

*Corresponding author's email: wisalbot@icp.edu.pk

Citation

Zahir Muhammad, Hazrat Ali, Wisal Muhammad Khan, Rehmanullah, Ghulam Jelani and Abdul Majeed.

Conservation status of plant resources of Hazar Nao hills, district Malakand, Pakistan. Pure and Applied Biology.

Vol. 7, Issue 3, pp931-945. http://dx.doi.org/10.19045/bspab.2018.700112

\begin{tabular}{llll}
\hline \hline Received: 15/03/2018 & Revised: 05/07/2018 & Accepted: 09/07/2018 & Online First: 18/07/2018
\end{tabular}

Abstract

In the present study, data collected for the conservation status following the International Union for Conservation of Nature (IUCN, 2001) dealt with 77 species that belonged to 42 families. It was noted that 20 species (25.97\%) were found to be endangered, 23 species $(29.87 \%)$ vulnerable, 15 species $(19.48 \%)$ rare and 19 species (24.67\%) infrequent. Due to overgrazing, excessive collection, burning and other anthropogenic activities the plant resources of Hazar Nao hills are under high pressure. Therefore, there is a dire need to establish a satisfactory in-situ conservation policy for the sustainable utilization of plant resources in the sampled region.

Keywords: Conservation; District Malakand; Hazar Nao hills; Plant resources; Pakistan

\section{Introduction}

Sustainability of forest resources is of utmost importance in the life of local communities as well as prevailing environmental conditions. Degradation of natural vegetation occurs due to unbalanced use of resources [1]. Deforestation and overgrazing are among the leading drivers which results in demised plant diversity and correspond to fragile ecosystems. Many plant species have either become threatened or endangered due to heavy pressure of anthropogenic activities, natural and managed overgrazing. Some forests have converted to scrubs and grasslands. Deforestation, overgrazing, uprooting of plants and terrace cultivation have not only accelerated soil erosion but decreased plant diversity in the area [2]. Pakistan, having less than $4 \%$ of its area covered by forest is further threatened by an annual deforestation rate of $3 \%$. More than $25 \%$ of Pakistani forests have been lost in last 3 decades [3]. Major cause of the current event is the habitat loss by various anthropogenic activities [4]. Plant extinction rate has reached to one species per day as a result of anthropogenic activities [3] and it is estimated that if no preventive measures are taken, 60,000-100,000 plant species are likely to disappear in the near future [4]. Plant preservation is a systemic approach towards the protection of biodiversity of plants by employing sustainable techniques [5]. In Pakistan, various workers have carried out 
conservation studies in different parts of the country. [6] showed that the vegetation cover of the established communities in Push Ziarat area is depleting at a very high pace due to population explosion. [7] determined the conservation status of 45 species belonging to 26 families, in Tehsil Takht-e-Nasrati. [8] determined the conservation status of 27 plant species belonging to 19 genera from different localities of District Mardan. [9] reported 43 threatened plant species from Dir Kohistan valley. [10] evaluated the conservation status of plant species in Tehsil Katlang. [11] carried out a research for biodiversity conservation in the Himalayan region of Poonch Valley. [12] carried out conservation studies of 45 threatened medicinal plants in Mankial valley. [13] documented the conservation status of plant species in Tehsil Takht Bhai. A thorough review of literature clearly depicted that no such study has been conducted on the Hazar Nao hills. The present work was therefore important to explore the conservation status of this remote area.

\section{Materials and methods}

\section{Study area}

Malakand lies $34^{0} 35 \mathrm{~N}$ latitude and $71^{0} 57 \mathrm{E}$ longitude. It is the gateway to Dir, Chitral, Swat Districts, Mohmand and Bajaur Agencies. It extends from Hindukush mountain range down to the northern edge of the Peshawar Basin. The total area extends over $952 \mathrm{Km}^{2}$ with a population density of 596 people per $\mathrm{Km}^{2}$. Malakand is 2705 feet above the sea level. The area is characterized by mountains. The climate of the area is dry. Hottest months are June, July and August. The maximum temperature during summer exceeds over $40^{\circ} \mathrm{C}$ and minimum in winter falls upto $6^{\circ} \mathrm{C}$. Malakand falls under subtropical zone hosting association of Dodonaea and Chir pine [14]. Hazar Nao hills are located at about 23 kilometers west of Tehsil Dargai, District Malakand [15]. The Hazar Nao hills are important mountains of Malakand district located between villages of Pir Khel, Mekhband, Totai, Kot, Maina, Brah, and Haryankot having about 2727 m altitude. The hills are thickly covered with forests and have its importance for botanical and medicinal plants studies (Figure 1) [16].

\section{Field survey}

The study was conducted by frequently surveying different sites of the research area. The area was divided into four sites viz; Kot, Maina, Brah,andTotai. Nature of habitat was analyzed by habitat destruction, soil erosion, fuelwood cutting, grazing, invasive species and impacts of other anthropogenic activities were noted [17].

\section{Plant collection and preservation}

Plant specimens were collected from different sites of the research area (Figure 313). Plants specimens were properly preserved and identified following [4]. The voucher specimens were deposited in the Herbarium, Department of Botany, University of Peshawar (UOP).

\section{Questionnaire development}

Information on demography (age, gender) and conservation status were gathered from each site by using a semi-structured questionnaire. The plants were classified according to their economic value through interviewing and filling questionnaires from drug dealers, shopkeepers, fuel wood seller, local hakims, and farmers but the priority was who were the real users and had a lot of information about the plants and their conservation status.

The data was classified, tabulated, analyzed and concluded for the final report. The conservation status of plants was determined according to IUCN [18], Red Data List Categories and Criteria.

The data was collected through following formula: $\mathrm{CSP}=\mathrm{A}+\mathrm{C}+\mathrm{G}+\mathrm{P}(\mathrm{Khan}, 2013)$. 


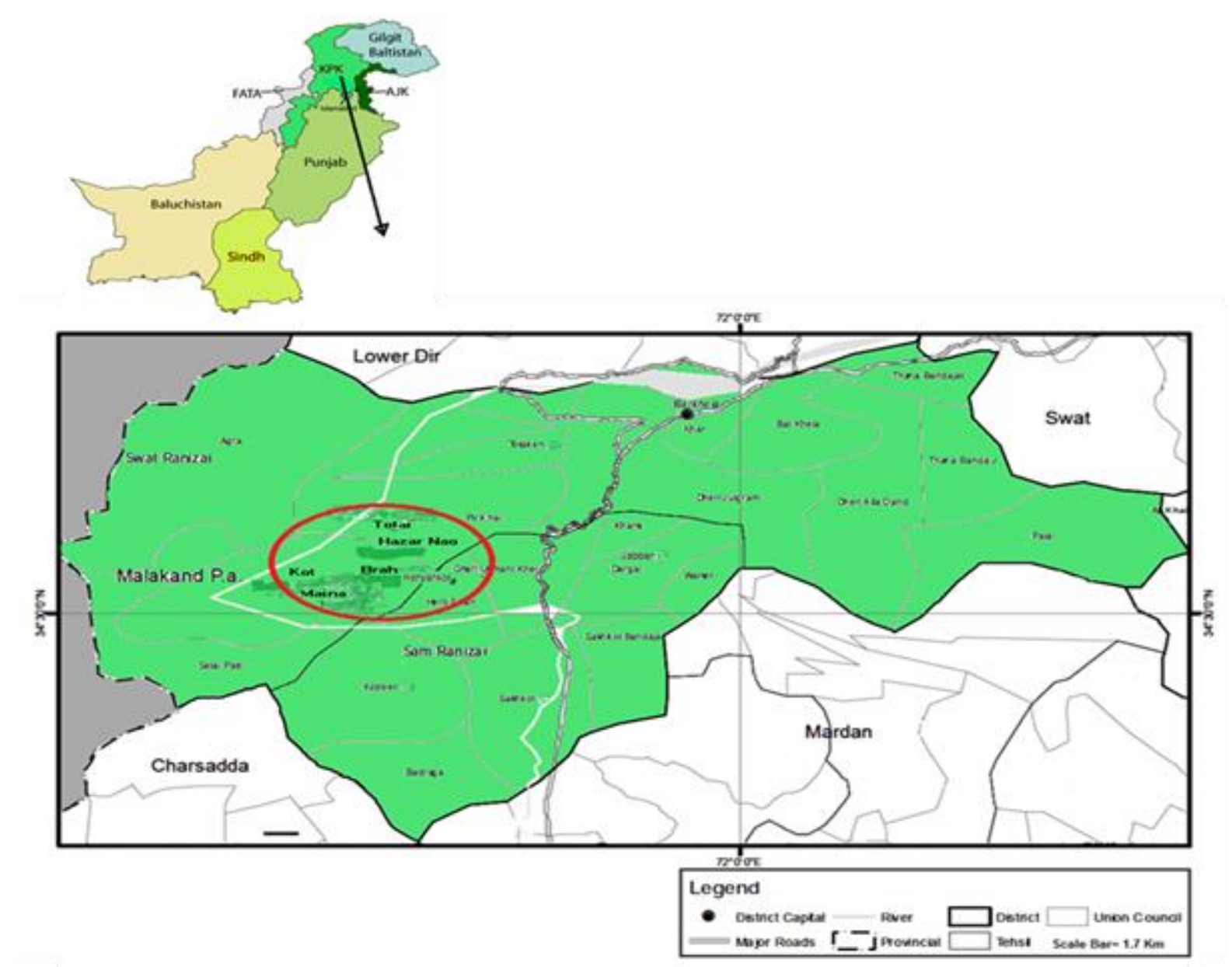

Figure 1. Map of the research area, district Malakand

\section{Results and discussion}

The data was collected for various conservation parameters following IUCN (2001). It was noted that 20 species $(25.97 \%)$ were found endangered, 23 plant species (29.87\%) vulnerable, 15 species $(19.48 \%)$ rare and 19 species $(24.67 \%)$ were infrequent (Figure 2). Due to the excessive collection, overgrazing and other human influences in the investigated areas of Hazar Nao hills, none of the species was declared as dominant which highlight the miserable current situation of plant resources in the area (Table 1).

\section{Endangered species}

In the present study, 20 species (25.97\%) were found endangered. The most important include Justicia adhatoda, Caralluma tuberculata, Quercus incana, Melia azedarach, Acacia modesta, Acacia nilotica, Albizia lebbeck, Ficus carica, Morus alba, Moru snigra, Olea ferruginea, Bauhinia variegata, Butea monosperma, (Figure 6) Dalbergia sissoo, Platanus orientalis, Ziziphus mauritiana, Ziziphus sativa, Salix tetrasperma, Monotheca buxifolia and Ailanthus altissima (Table 1). Endangered species in different parts of Pakistan were also reported by $[5,13,19]$. These plants were used for medicinal purposes, fuelwood (Figure 8), timber wood, furniture and agriculture, coupled with harsh climatic and habitat condition which has slow down their natural regeneration. Over-harvesting, destruction of habitat (Figure 10), population growth, urbanization and the unrestricted collection of medicinal plants from the wild 
results in over-exploitation of natural resources [20].

\section{Vulnerable species}

Twenty-three plant species $(29.87 \%)$ were vulnerable (Table 1). Plants like Maytenus royleanus, Vitex negundo, Ziziphus nummularia etc are intensively used as hedge. These have low regeneration capacity. Aridity of area and extensive clipping has pushed them into vulnerable categories. Colebrookea oppositifolia, Mallotus phillipensis, Ricinus communis, Vitis jacquemontii, Nerium oleander, Otostegia limbata, Periploca aphylla, Rhazya stricta, Xanthium strumarium and Dodonae aviscosa are fuel wood species. Chrysopogon aucheri, Saccharum griffithii and Indigofera heterantha become vulnerable due to burning (Figure 5), grazing (Figure 4) and fodder use for livestock. Ficus bengalensis, Myrtus communis, Phoenix sylvestris, Punica granatum, Pyrus pashia and Sageretia thea are used for wild fruits, fuel, fodder etc. so become vulnerable. Some plants are used for medicinal purposes. Over collection as medicinal material and lack of suitable habitat has hampered their regeneration leading them to vulnerable category. Earlier researchers also recorded vulnerable species in their studied areas $[3,10,14]$.

\section{Rare species}

The present study explored 15 (19.48\%) species rare (Table 1). Plants such as Chrysopogon aucheri, Chrysopogon serrulatus, Saccharum griffithii, Calotropis procera, Cannabis sativa, Mentha arvensis, Mentha longifolia, Woodfordia fruticosa, Indigofera heterantha, Plantago lanceolata, Rubus fruticosus, Verbascum thapsus, Hyoscyamus insanus and Vitis jacquemontii were used for various purposes like fuel wood, fodder/forage and as health care medicines, leading them to rare category. [8] carried out conservation assessment in the remote Hindukush mountain region of District Swat. The study revealed that $49 \%$ of these economically valued medicinal plant species were threatened due to increased exploitation and unsustainable harvesting. [20] observed that the rare and threatened species of MAPs were collected in a highly unsustainable manner, therefore causing biodiversity loss and depletion of MAPs population.

Table 1. Ranking of species using IUCN criteria (2001) for conservation status of plants of Hazar Nao forest, district Malakand

\begin{tabular}{|c|c|c|c|c|c|c|c|}
\hline No & Divivision/Family/ Species & $\begin{array}{c}\text { Availability } \\
\text { class }\end{array}$ & $\begin{array}{c}\text { Collection } \\
\text { status }\end{array}$ & $\begin{array}{l}\text { Part } \\
\text { used }\end{array}$ & $\begin{array}{c}\text { Growth } \\
\text { behavior }\end{array}$ & $\begin{array}{l}\text { Total } \\
\text { score }\end{array}$ & $\begin{array}{c}\text { Conservation } \\
\text { status }\end{array}$ \\
\hline $\mathbf{A}$ & PTERIDOPHYTA & & & & & & \\
\hline 1 & Adiantaceae & & & & & & \\
\hline 1 & Adiantum capillus-veneris $\mathrm{L}$. & 1 & 3 & 4 & 4 & 12 & Rare \\
\hline $\mathbf{B}$ & GYMNOSPERMS & & & & & & \\
\hline 2 & Pinaceae & & & & & & \\
\hline 2 & Pinus roxburghii Sargent & 3 & 2 & 1 & 0 & 6 & Vulnerable \\
\hline $\mathrm{C}$ & MONOCOTS & & & & & & \\
\hline 3 & Palmae & & & & & & \\
\hline 3 & $\begin{array}{l}\text { Nannorrhop sritchiana (Griff.) } \\
\text { Aitchison. }\end{array}$ & 0 & 1 & 1 & 4 & 6 & Vulnerable \\
\hline 4 & Phoenix sylvestris (L.) Roxb. & 1 & 3 & 0 & 2 & 6 & Vulnerable \\
\hline 4 & Poaceae & & & & & & \\
\hline 5 & Chrysopogon aucheri (Boiss.) & 3 & 3 & 0 & 4 & 10 & Rare \\
\hline 6 & C. serrulatusTrin. & 3 & 3 & 0 & 4 & 10 & Rare \\
\hline 7 & C. jwarancusa(Jones) Schult. & 3 & 3 & 4 & 4 & 14 & Infrequent \\
\hline 8 & Cynodon dactylon (L.) Pers. & 3 & 3 & 4 & 4 & 14 & Infrequent \\
\hline 9 & $\begin{array}{l}\text { Heteropogon contortus (L.) } \\
\text { Beauv. }\end{array}$ & 3 & 3 & 4 & 4 & 14 & Infrequent \\
\hline
\end{tabular}




\begin{tabular}{|c|c|c|c|c|c|c|c|}
\hline 10 & $\begin{array}{l}\text { Saccharum griffithii Munro ex } \\
\text { Boiss. }\end{array}$ & 3 & 1 & 2 & 4 & 10 & Rare \\
\hline D & DICOTS & & & & & & \\
\hline 5 & Acanthaceae & & & & & & \\
\hline 11 & Justicia adhatoda L. & 1 & 1 & 2 & 0 & 4 & Endangered \\
\hline 6 & Amaranthaceae & & & & & & \\
\hline 12 & Achyranthes aspera L. & 3 & 3 & 4 & 4 & 14 & Infrequent \\
\hline 13 & Amaranthus caudatus L. & 3 & 3 & 4 & 4 & 14 & Infrequent \\
\hline 7 & Apocynaceae & & & & & & \\
\hline 14 & Nerium oleander $\mathrm{L}$. & 1 & 3 & 3 & 1 & 8 & Vulnerable \\
\hline 15 & Rhazya stricta Decne. & 1 & 2 & 3 & 0 & 6 & Vulnerable \\
\hline 8 & Asclepiadaceae & & & & & & \\
\hline 16 & Calotropis procera (Willd) R.Br. & 1 & 3 & 3 & 4 & 11 & Rare \\
\hline 17 & $\begin{array}{l}\text { Caralluma tuberculate N. E. } \\
\text { Brown }\end{array}$ & 0 & 2 & 1 & 1 & 4 & Endangered \\
\hline 18 & Periploca aphyllaDcne. & 2 & 2 & 3 & 1 & 8 & Vulnerable \\
\hline 9 & Asteraceae & & & & & & \\
\hline 19 & $\begin{array}{lll}\text { Launaea procumbens } & \text { (Roxb.) } \\
\text { Ramayya \&Rajagopal } & \\
\end{array}$ & 3 & 3 & 4 & 4 & 14 & Infrequent \\
\hline 20 & Carthamus oxycantha M. B. & 3 & 3 & 4 & 4 & 14 & Infrequent \\
\hline 21 & Xanthium strumarium L. & 1 & 3 & 3 & 0 & 7 & Vulnerable \\
\hline 10 & Berberidaceae & & & & & & \\
\hline 22 & Berberis lycium Royle & 3 & 3 & 1 & 1 & 8 & Vulnerable \\
\hline 11 & Canabanaceae & & & & & & \\
\hline 23 & Cannabis sativa $\mathrm{L}$. & 1 & 3 & 4 & 4 & 12 & Rare \\
\hline 12 & Celastraceae & & & & & & \\
\hline 24 & $\begin{array}{l}\text { Maytenus royleanus (Wall. ex } \\
\text { Lawson) Cuf. }\end{array}$ & 1 & 2 & 2 & 1 & 6 & Vulnerable \\
\hline 13 & Chenopodiaceae & & & & & & \\
\hline 25 & Chenopodium album $\mathrm{L}$. & 3 & 3 & 4 & 4 & 14 & Infrequent \\
\hline 14 & Convolvulaceae & & & & & & \\
\hline 26 & Convolvulus arvensis L. & 3 & 3 & 4 & 4 & 14 & Infrequent \\
\hline 15 & Euphorbiaceae & & & & & & \\
\hline 27 & Euphorbia helioscopia L. & 3 & 3 & 4 & 4 & 14 & Infrequent \\
\hline 28 & $\begin{array}{l}\begin{array}{l}\text { Mallotus phillipensis (Lam) } \\
\text { Muell. }\end{array} \\
\text { la }\end{array}$ & 1 & 1 & 3 & 1 & 6 & Vulnerable \\
\hline 29 & Phyllanthus emblica $\mathrm{L}$. & 1 & 3 & 1 & 2 & 7 & Vulnerable \\
\hline 30 & Ricinus communis $\mathrm{L}$. & 1 & 3 & 1 & 0 & 5 & Vulnerable \\
\hline 16 & Fagaceae & & & & & & \\
\hline 31 & Quercus incanaRoxb. & 0 & 1 & 1 & 1 & 3 & Endangered \\
\hline 17 & Fumariaceae & & & & & & \\
\hline 32 & $\begin{array}{l}\text { Fumaria indica } \\
\text { Pugsley }\end{array}$ & 3 & 3 & 4 & 4 & 14 & Infrequent \\
\hline 18 & Lamiaceae & & & & & & \\
\hline 33 & Ajuga bracteosa Wall. Ex Benth. & 3 & 3 & 4 & 4 & 14 & Infrequent \\
\hline 34 & Colebrookea oppositifolia Smith & 1 & 2 & 2 & 1 & 6 & Vulnerable \\
\hline 35 & Mentha arvensis L. & 2 & 3 & 3 & 4 & 12 & Rare \\
\hline 36 & Mentha longifolia $\mathrm{L}$ & 2 & 3 & 3 & 4 & 12 & Rare \\
\hline 37 & Otostegia limbata (Bth.) Boiss. & 4 & 2 & 2 & 0 & 8 & Vulnerable \\
\hline 19 & Lythraceae & & & & & & \\
\hline 38 & Woodfordia fruticosa $(\mathrm{L})$ Kurz & 0 & 3 & 3 & 4 & 10 & Rare \\
\hline 20 & Malvaceae & & & & & & \\
\hline 39 & Malva neglecta Wall. & 3 & 3 & 4 & 4 & 14 & Infrequent \\
\hline 21 & Meliaceae & & & & & & \\
\hline
\end{tabular}


Muhammad et al.

\begin{tabular}{|c|c|c|c|c|c|c|c|}
\hline 40 & Melia azedarach $\mathrm{L}$. & 0 & 1 & 1 & 1 & 3 & Endangered \\
\hline 22 & Mimosaceae & & & & & & \\
\hline 41 & Acacia modesta Wall. & 1 & 1 & 1 & 0 & 3 & Endangered \\
\hline 42 & A. nilotica(L.) Delile & 1 & 2 & 1 & 0 & 4 & Endangered \\
\hline 43 & Albizia lebbeck (L.) Benth. & 0 & 2 & 1 & 0 & 3 & Endangered \\
\hline 23 & Moraceae & & & & & & \\
\hline 44 & Ficus carica Hausskn. Ex. Boiss. & 1 & 2 & 1 & 0 & 4 & Endangered \\
\hline 45 & F. benghalensis L. & 1 & 2 & 1 & 1 & 5 & Vulnerable \\
\hline 46 & Morus alba $\mathrm{L}$. & 0 & 1 & 2 & 0 & 3 & Endangered \\
\hline 47 & M. nigra L. & 0 & 1 & 2 & 0 & 3 & Endangered \\
\hline 24 & Myrtaceae & & & & & & \\
\hline 48 & $\begin{array}{ll}\text { Eucalyptus } & \text { camaldulensis } \\
\text { Schlecht } & \\
\end{array}$ & 3 & 0 & 3 & 0 & 6 & Vulnerable \\
\hline 49 & Myrtus communis $\mathrm{L}$. & 0 & 2 & 3 & 2 & 7 & Vulnerable \\
\hline 25 & Nyctaginaceae & & & & & & \\
\hline 50 & $\begin{array}{l}\text { Boerhavia procumbens Banks ex } \\
\text { Roxb. }\end{array}$ & 3 & 3 & 4 & 4 & 14 & Infrequent \\
\hline 26 & Oleaceae & & & & & & \\
\hline 51 & Olea ferruginea Royle & 0 & 2 & 1 & 0 & 3 & Endangered \\
\hline 27 & Oxalidaceae & & & & & & \\
\hline 52 & Oxalis corniculata $\mathrm{L}$. & 3 & 3 & 4 & 4 & 14 & Infrequent \\
\hline 28 & Papilionaceae & & & & & & \\
\hline 53 & Bauhinia variegata $\mathrm{L}$. & 0 & 2 & 1 & 0 & 3 & Endangered \\
\hline 54 & Butea monosperma Lam. Taub. & 0 & 2 & 1 & 1 & 4 & Endangered \\
\hline 55 & Dalbergia sissoo Roxb. & 1 & 1 & 1 & 0 & 3 & Endangered \\
\hline 56 & $\begin{array}{l}\text { Indigofera heterantha Wall. ex } \\
\text { Brand. }\end{array}$ & 1 & 3 & 3 & 4 & 11 & Rare \\
\hline 29 & Plantaginaceae & & & & & & \\
\hline 57 & Plantago lanceolata $\mathrm{L}$. & 2 & 3 & 3 & 4 & 12 & Rare \\
\hline 58 & Platanus orientalis L. & 0 & 2 & 1 & 0 & 3 & Endangered \\
\hline 30 & Polygonaceae & & & & & & \\
\hline 59 & Rumex hastatus D. Don & 3 & 3 & 4 & 4 & 14 & Infrequent \\
\hline 31 & Punicaceae & & & & & & \\
\hline 60 & Punica granatumL. & 0 & 3 & 1 & 2 & 6 & Vulnerable \\
\hline 32 & Rhamnaceae & & & & & & \\
\hline 61 & $\begin{array}{lll}\begin{array}{l}\text { Sageretia } \\
\text { Jhonston }\end{array} & & \text { M.C. } \\
\end{array}$ & 1 & 3 & 1 & 2 & 7 & Vulnerable \\
\hline 62 & Ziziphus mauritiana Lam. & 0 & 2 & 1 & 0 & 3 & Endangered \\
\hline 63 & Z. sativa Gaertn. & 0 & 2 & 1 & 0 & 3 & Endangered \\
\hline 64 & $\begin{array}{l}\text { Z. nummularia (Burm. f.) Wight } \\
\text { \&Arn. }\end{array}$ & 1 & 3 & 2 & 0 & 6 & Vulnerable \\
\hline 33 & Rosaceae & & & & & & \\
\hline 65 & Pyrus pashia Ham. ex D. Don & 0 & 3 & 1 & 2 & 6 & Vulnerable \\
\hline 66 & Rubus fruticosus Wallich & 1 & 3 & 3 & 2 & 9 & Rare \\
\hline 34 & Salicaceae & & & & & & \\
\hline 67 & Salix tetrasperma Roxb. & 0 & 1 & 1 & 0 & 2 & Endangered \\
\hline 35 & Sapindaceae & & & & & & \\
\hline 68 & Dodonaea viscosa (L.) Jacq. & 3 & 0 & 3 & 0 & 6 & Vulnerable \\
\hline 36 & Sapotaceae & & & & & & \\
\hline 69 & $\begin{array}{l}\text { Monotheca buxifolia (Falc.) A. } \\
\text { DC. }\end{array}$ & 1 & 2 & 1 & 0 & 4 & Endangered \\
\hline 37 & Scrophulariaceae & & & & & & \\
\hline 70 & Verbascum Thapsus L. & 1 & 3 & 2 & 4 & 10 & Rare \\
\hline
\end{tabular}




\begin{tabular}{|c|c|c|c|c|c|c|c|}
\hline 38 & Simarubaceae & & & & & & \\
\hline 71 & $\begin{array}{lll}\begin{array}{l}\text { Ailanthus altissima } \\
\text { Swingle }\end{array} & \text { (Mill.) } \\
\end{array}$ & 0 & 1 & 3 & 0 & 4 & Endangered \\
\hline 39 & Solanaceae & & & & & & \\
\hline 72 & Hyoscyamus insanus Stocks & 0 & 3 & 4 & 4 & 11 & Rare \\
\hline 73 & Solanum nigrumL. & 3 & 3 & 4 & 4 & 14 & Infrequent \\
\hline 74 & Solanum surattense Burm. f. & 3 & 3 & 4 & 4 & 14 & Infrequent \\
\hline 40 & Verbenaceae & & & & & & \\
\hline 75 & Vitex negundo $\mathrm{L}$. & 1 & 3 & 3 & 0 & 7 & Vulnerable \\
\hline 41 & Vitaceae & & & & & & \\
\hline 76 & Vitis jacquemontii R. Parker & 1 & 2 & 3 & 4 & 10 & Rare \\
\hline 42 & Zygophyllaceae & & & & & & \\
\hline 77 & Tribulus terrestris L. & 3 & 3 & 4 & 4 & 14 & Infrequent \\
\hline
\end{tabular}

Key:

1. Availability

$0=$ Uncommon or very rare

$1=$ Less common or rare

$2=$ Occasional

$3=$ Abundant

\section{Collection}

$0=$ More than $1000 \mathrm{~kg} / \mathrm{yr}$

$1=$ Consumed from $500-1000 \mathrm{~kg} / \mathrm{yr}$

$2=$ Consumed from $300-500 \mathrm{~kg} / \mathrm{yr}$

3 = Consumed from $100-200 \mathrm{~kg} / \mathrm{yr}$

\section{Growth}

$0=$ Regrowth in more 3 years

$1=$ Regrowth within 3 years

$2=$ Regrowth within 2 years
$3=$ Regrowth within 1 year

4 = Leaves/Gum/Latex

4. Part used

$0=$ Root $/$ Whole plant

$1=$ Bark

$2=$ Seeds, Fruits

$3=$ Flowers

4 = Leaves/Gum/Latex

\section{Total Score}

6. 0 - 4 Endangered

7. 5 - 8 Vulnerable

8. 9 - 12 Rare:

9. 13 - 14 Infrequent

10. 15 - 16 Dominant
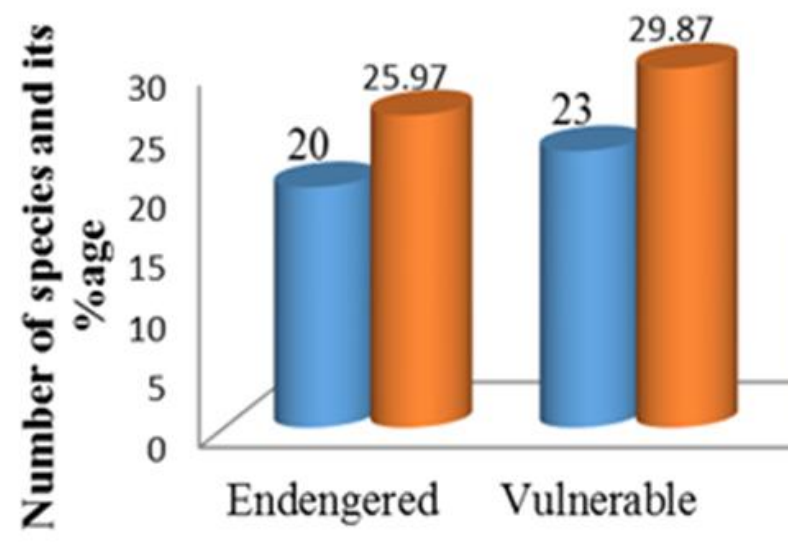

number of species $\quad$ N Percentage

Figure 2. Conservation ststus of plant resources of Hazar Nao hills, district Malakand 


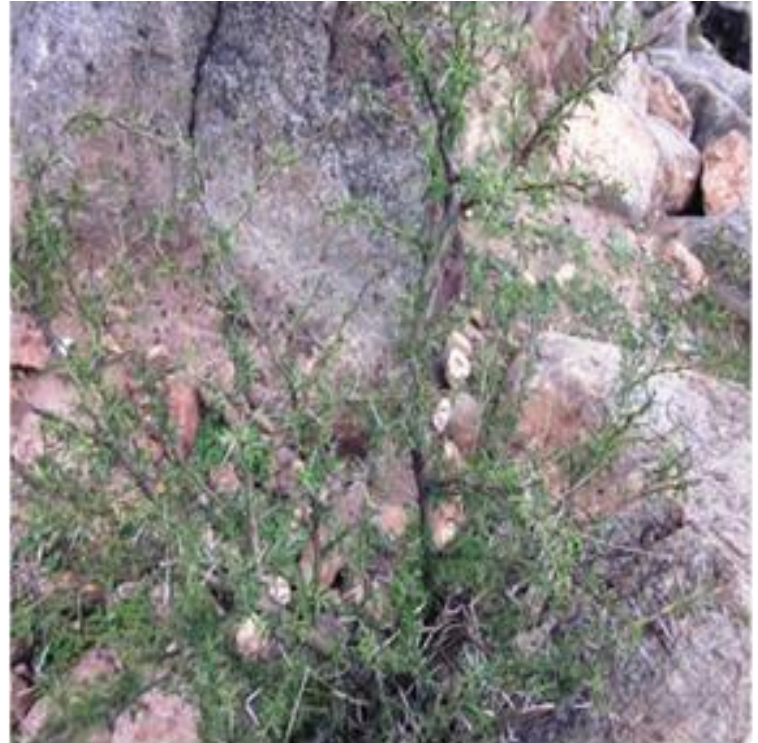

Acacia nelotica

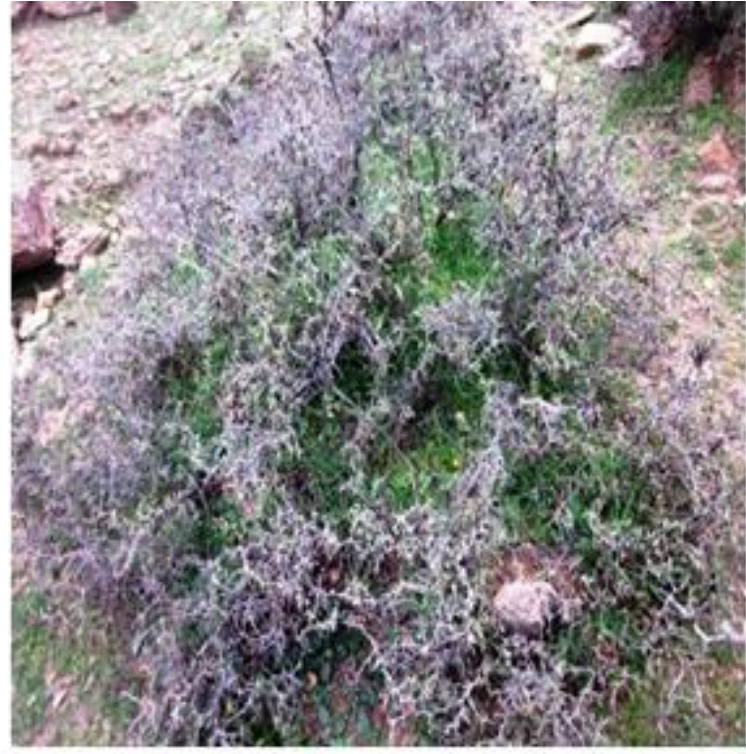

Ziziphus nummularia

Figure 3. Bushy appearance of Acacia nelotica and Ziziphus nummularia after grazing

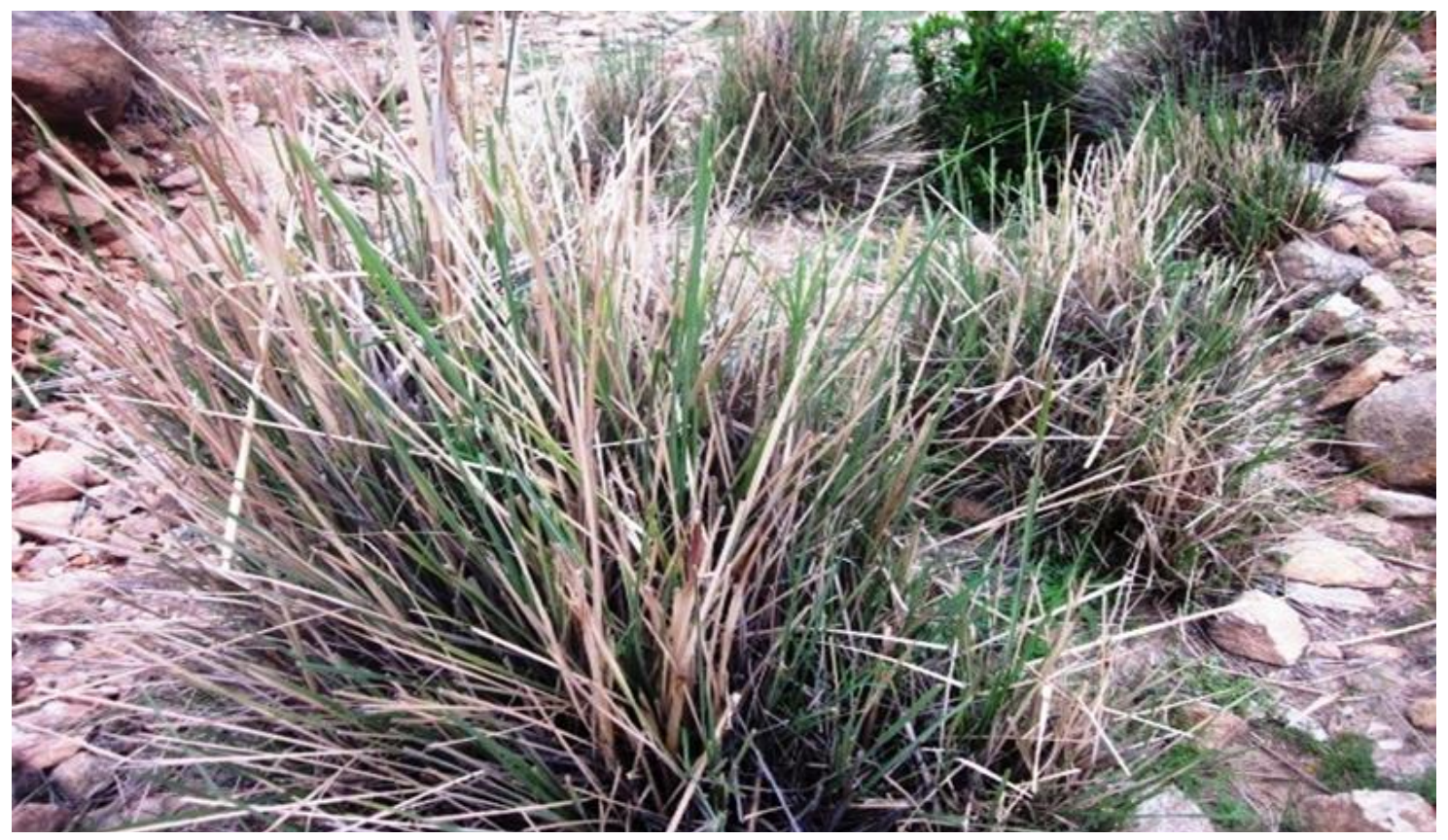

Figure 4. Heavy grazing caused bushy appearance of Saccharum griffithii 


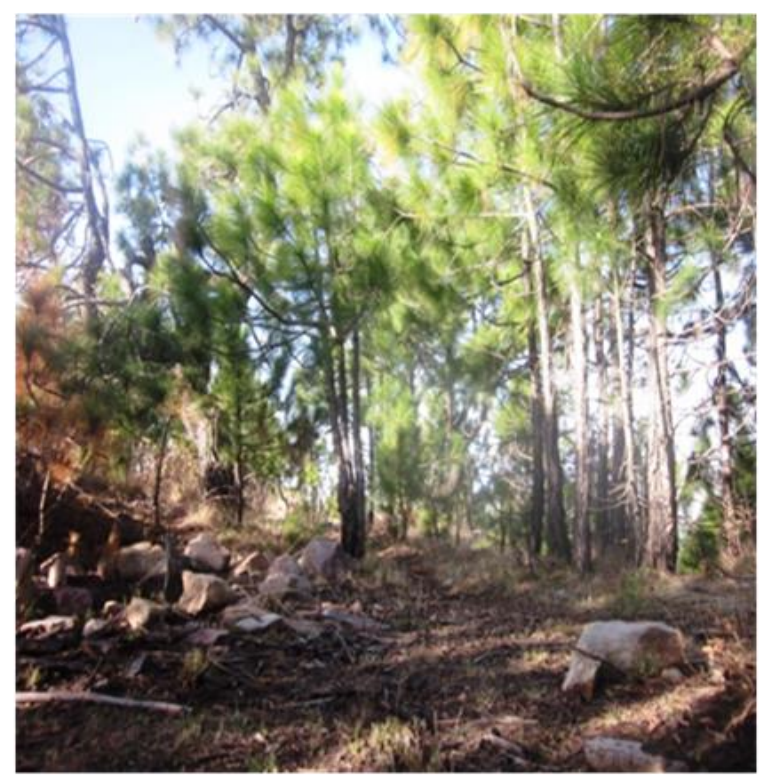

A

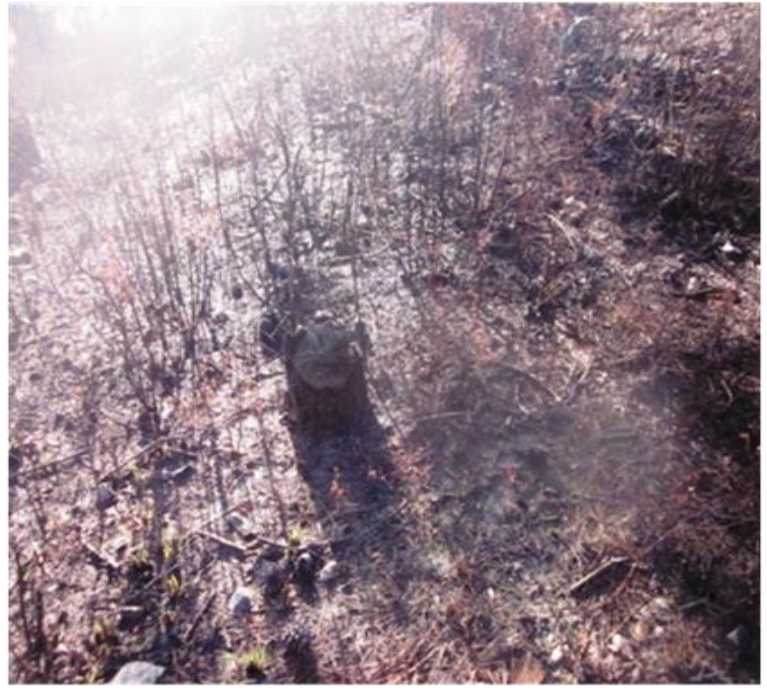

$\mathrm{C}$

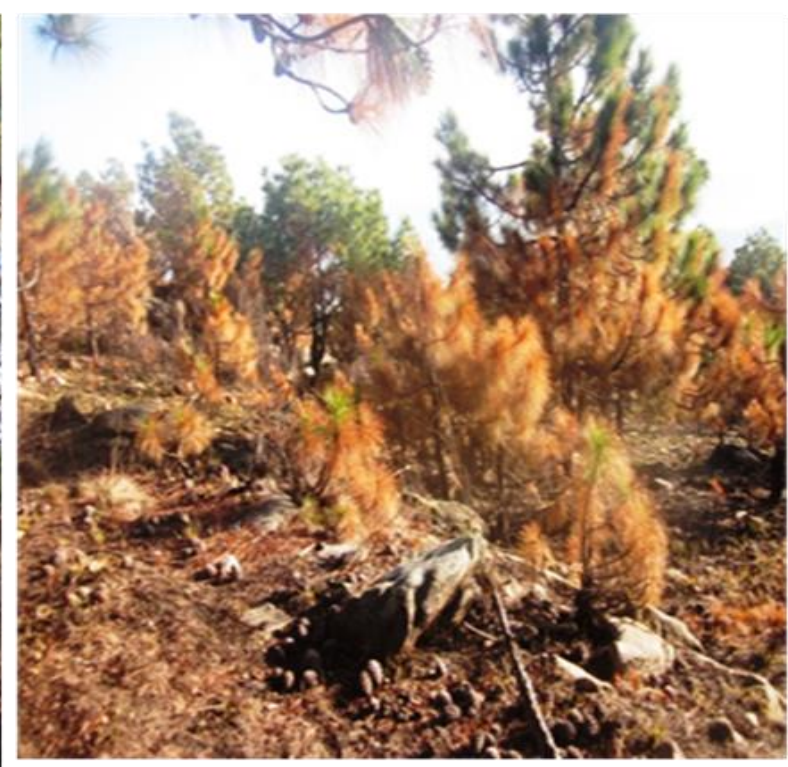

B

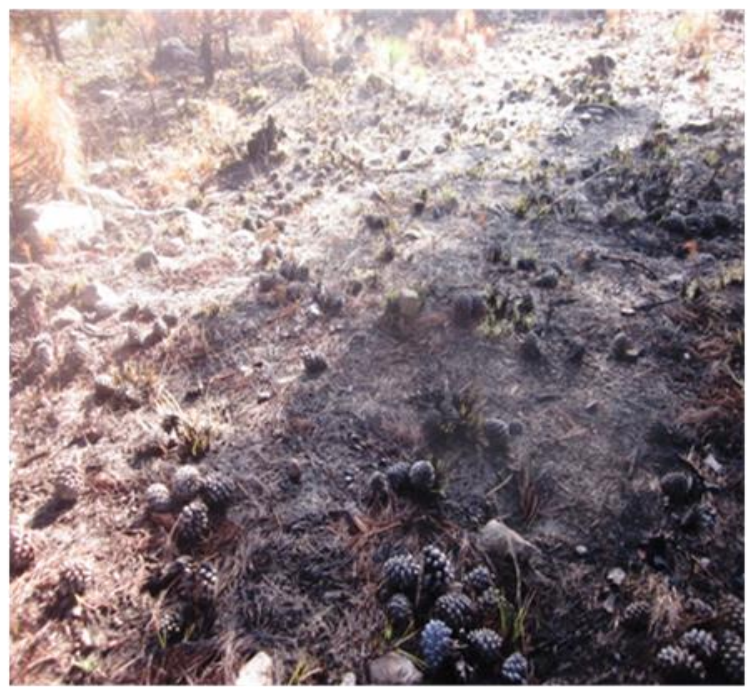

D

Figure 5. (A, B, C, D) Fire an ecological factor clears the green belt of vegetation 


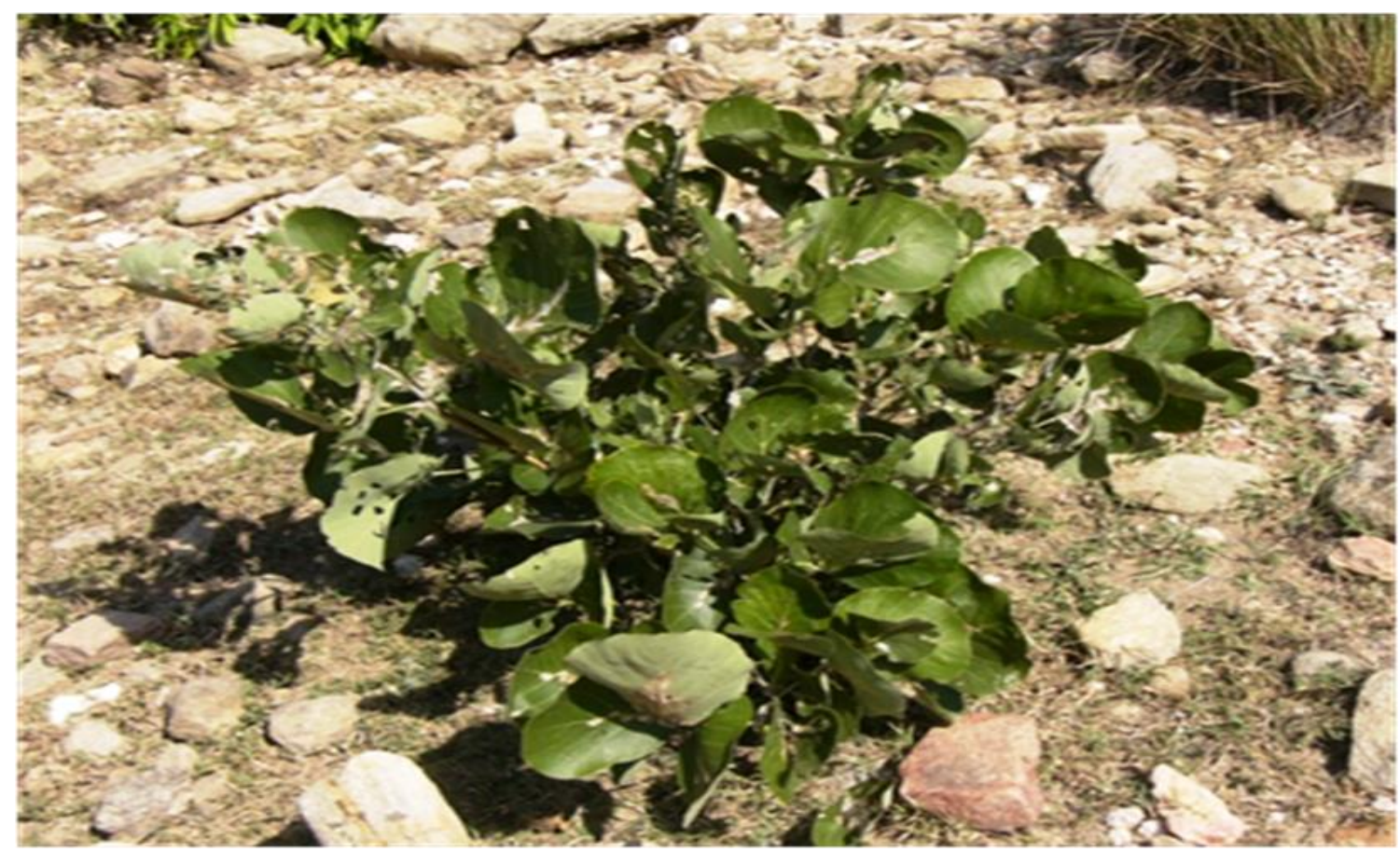

Figure 6. A view of degraded plant of Butea monosperma

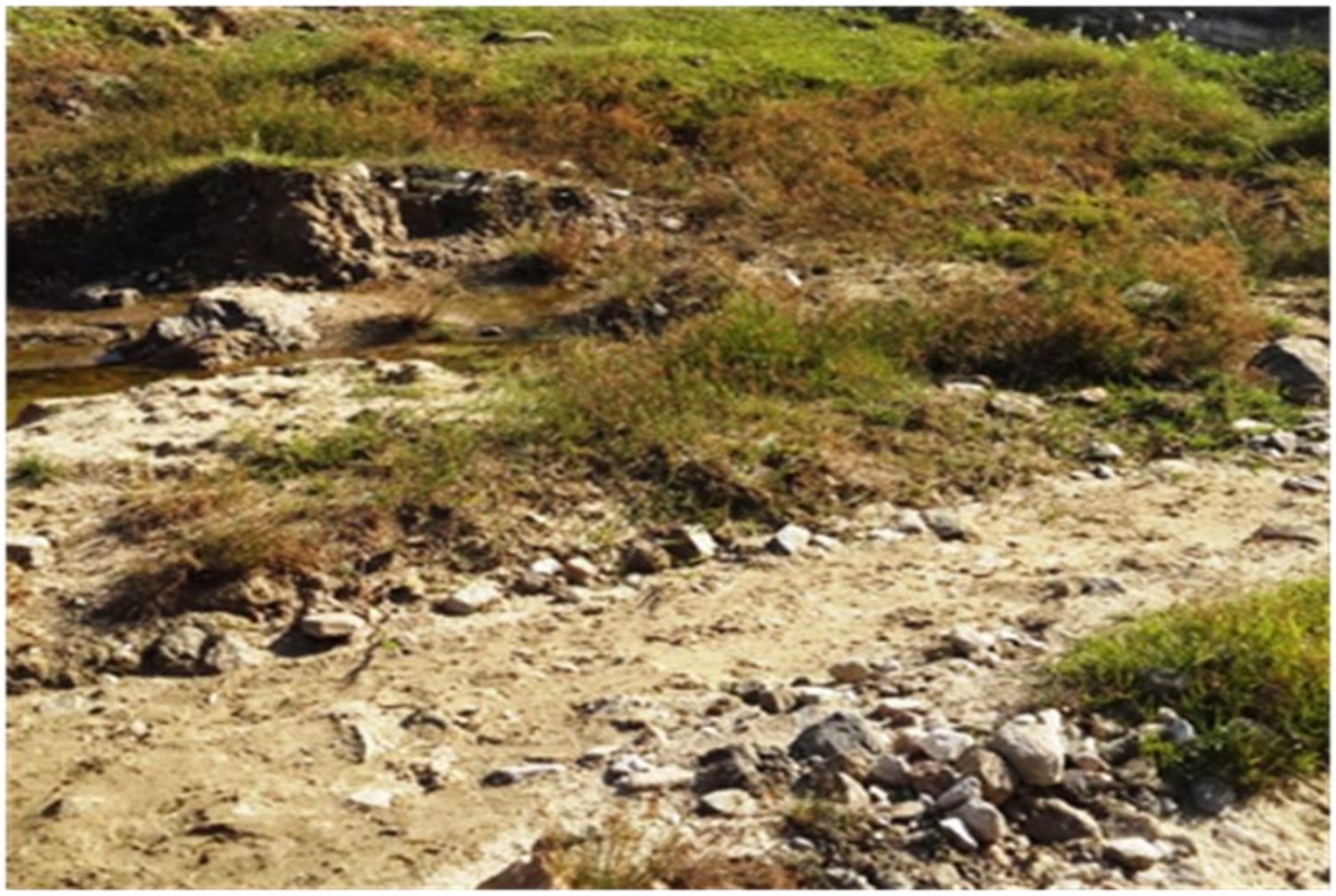

Figure 7. Soil erosion in the study area 


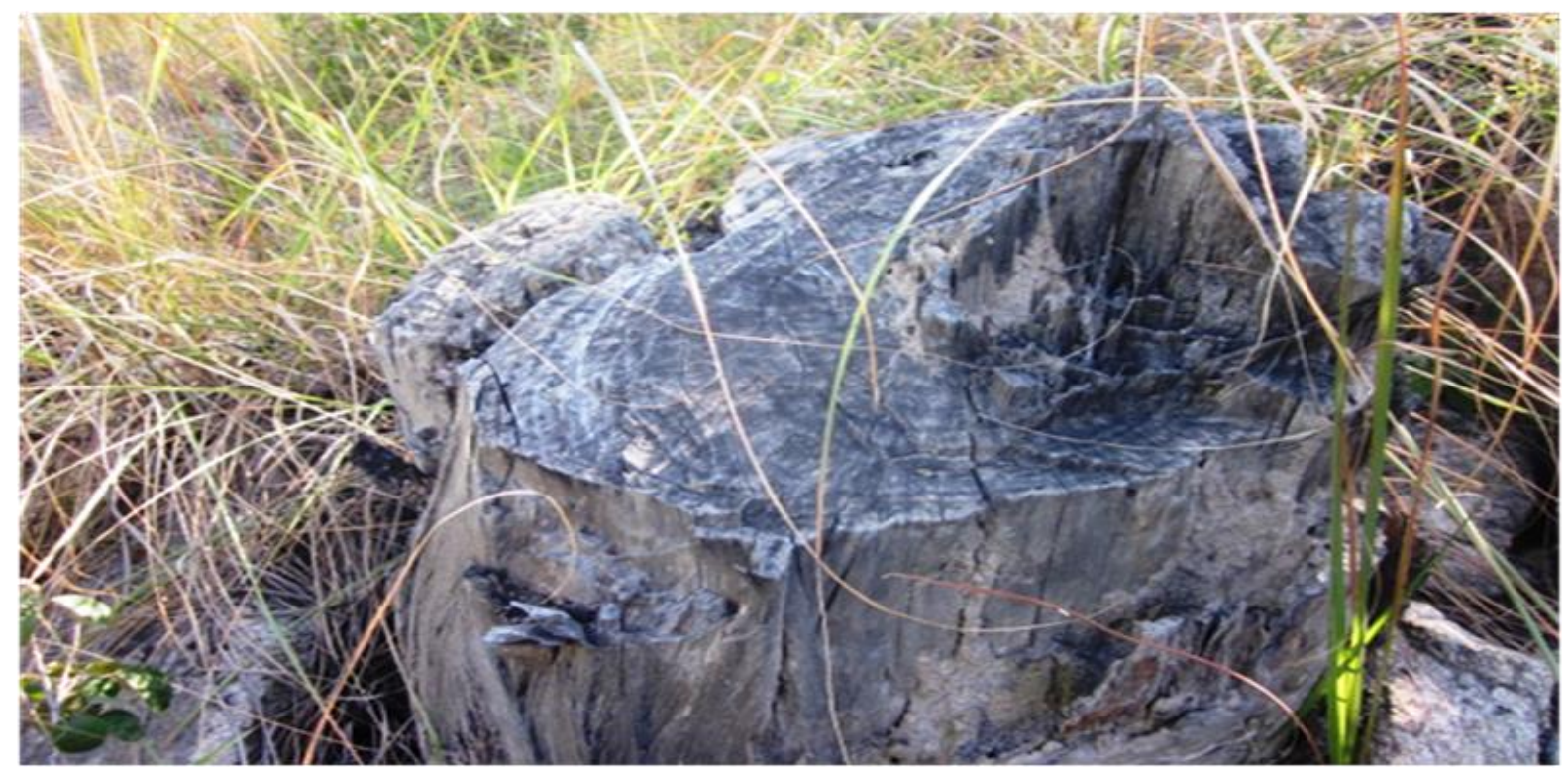

Figure 8. Cutting of trees is a common practice leading to low species diversity and maturity

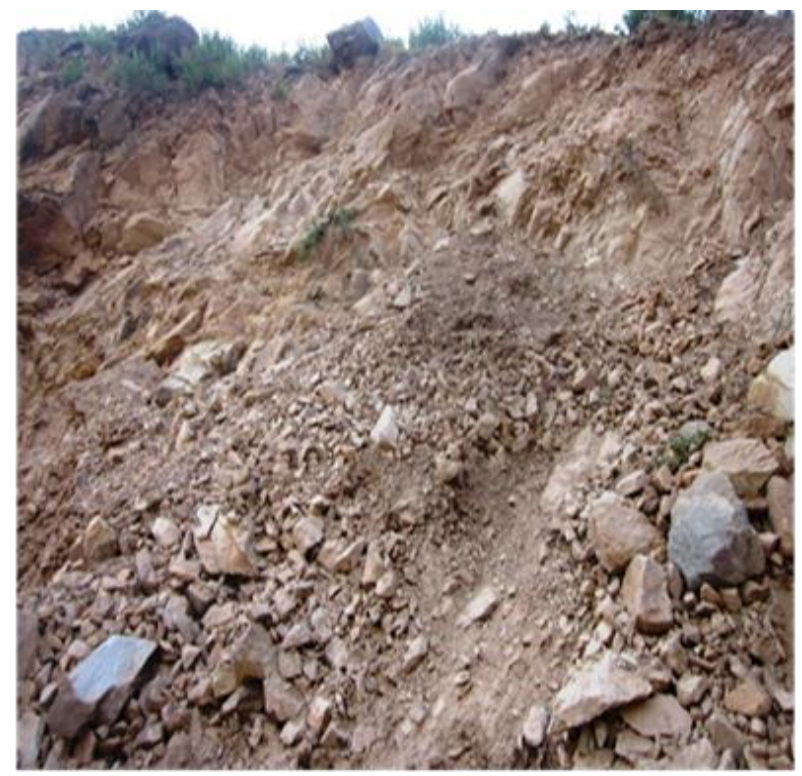

A

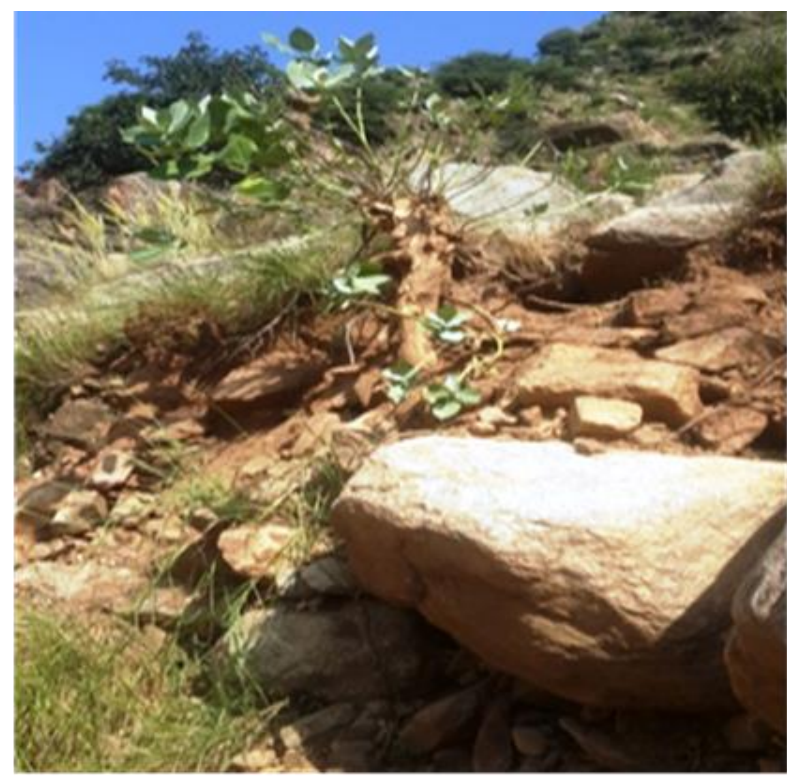

B

Figure 9. (A-B) Land sliding cause degradation of vegetation 


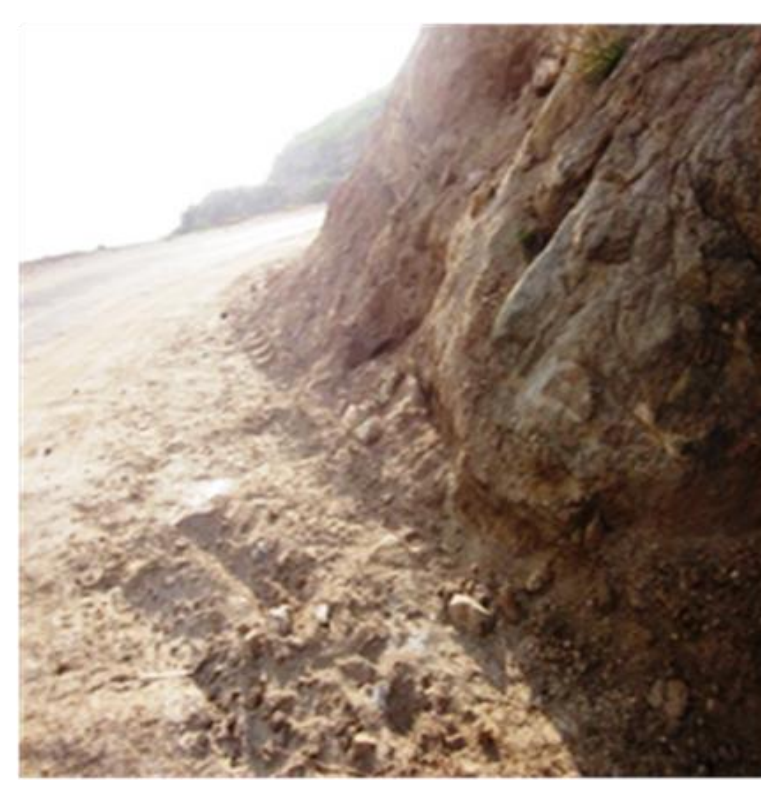

A

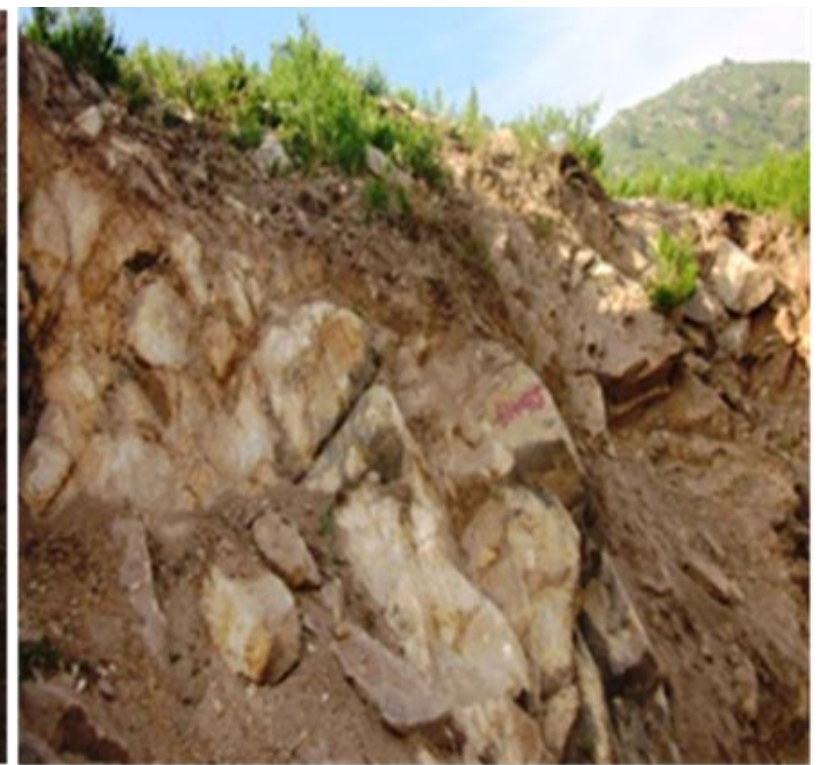

B

Figure 10. (A, B) Road expansion is a threat to plant resources

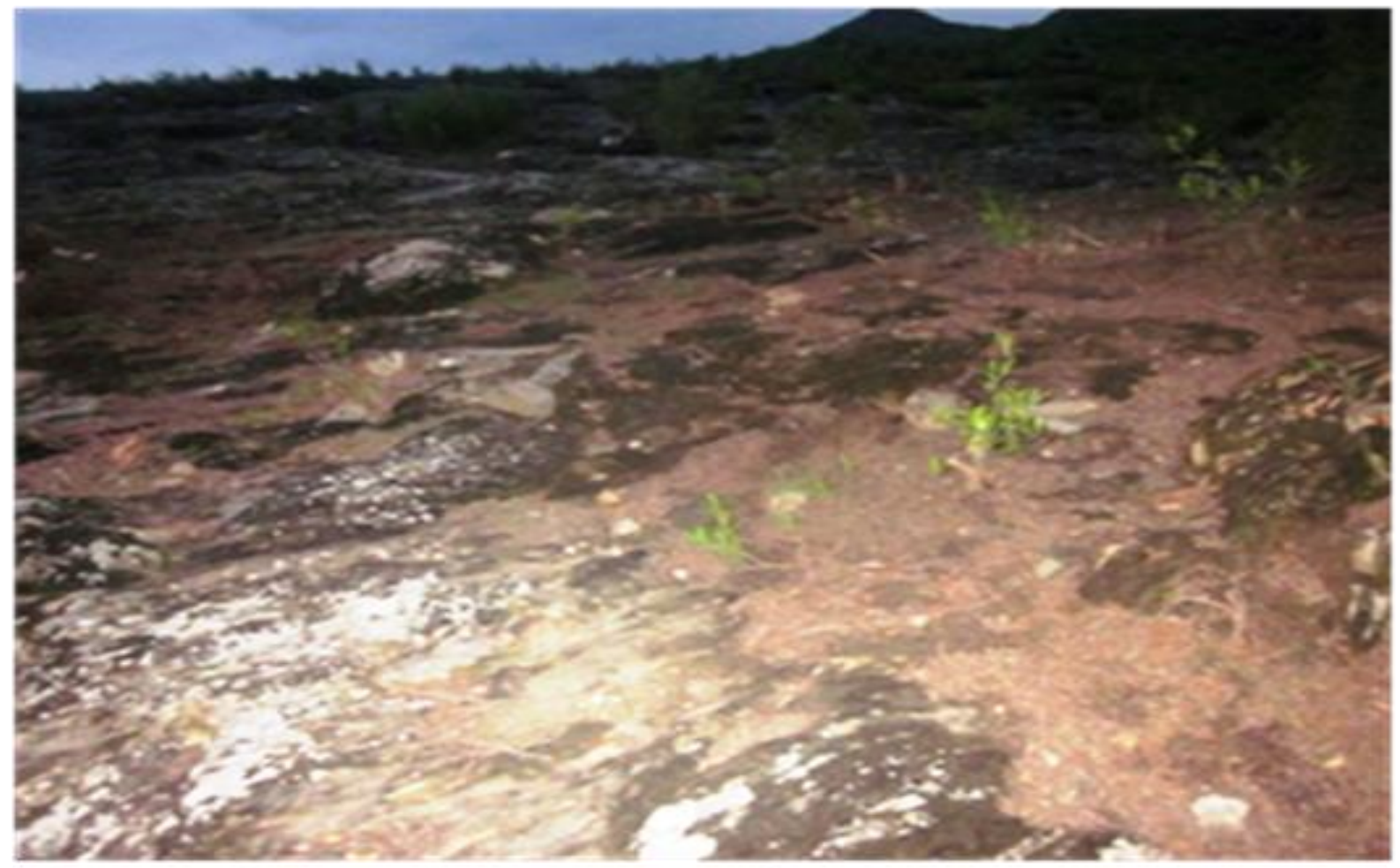

Figure 11. Barren area emerged due to fuel wood cutting 


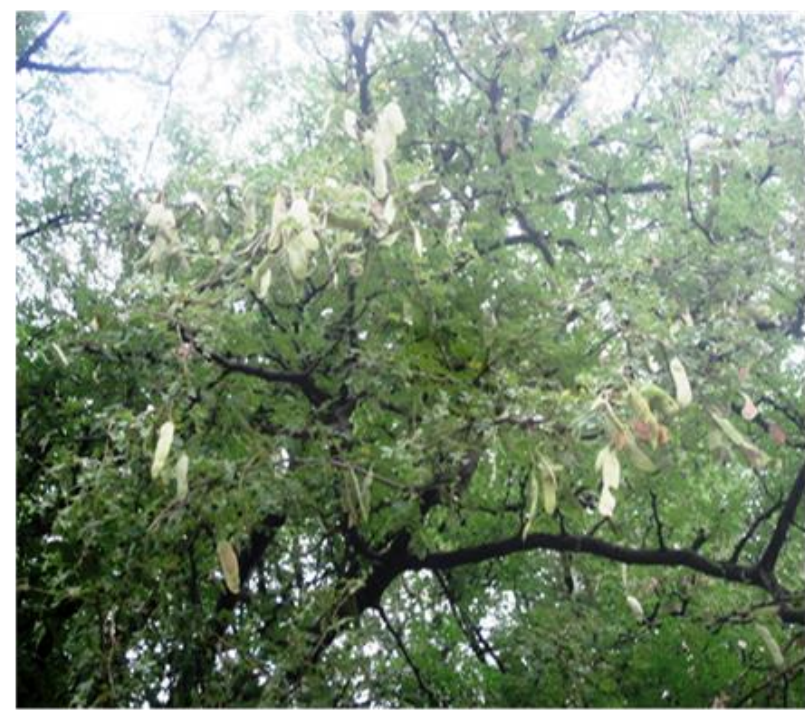

A

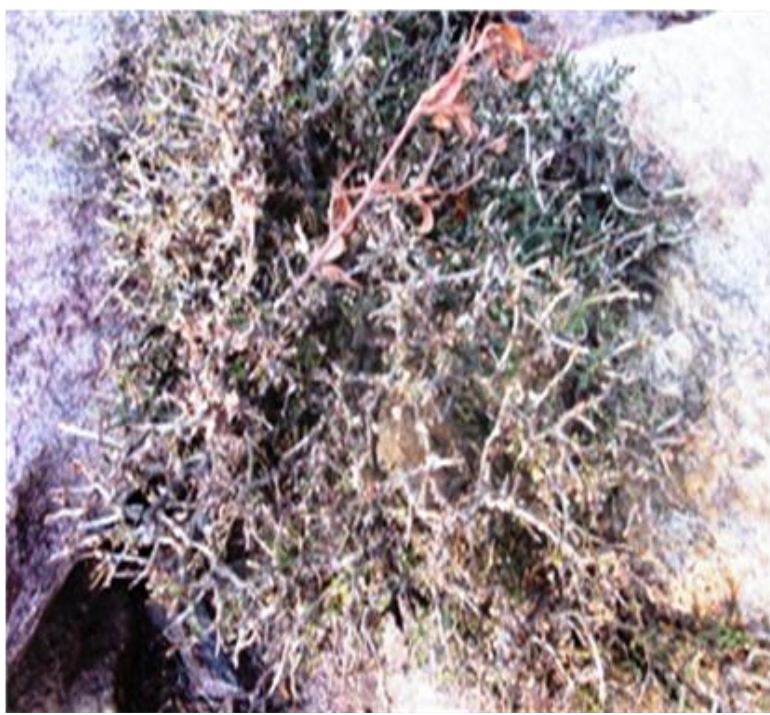

B

Figure 12. (A) Mature Acacia modesta adopted bushy appearance after heavily grazed

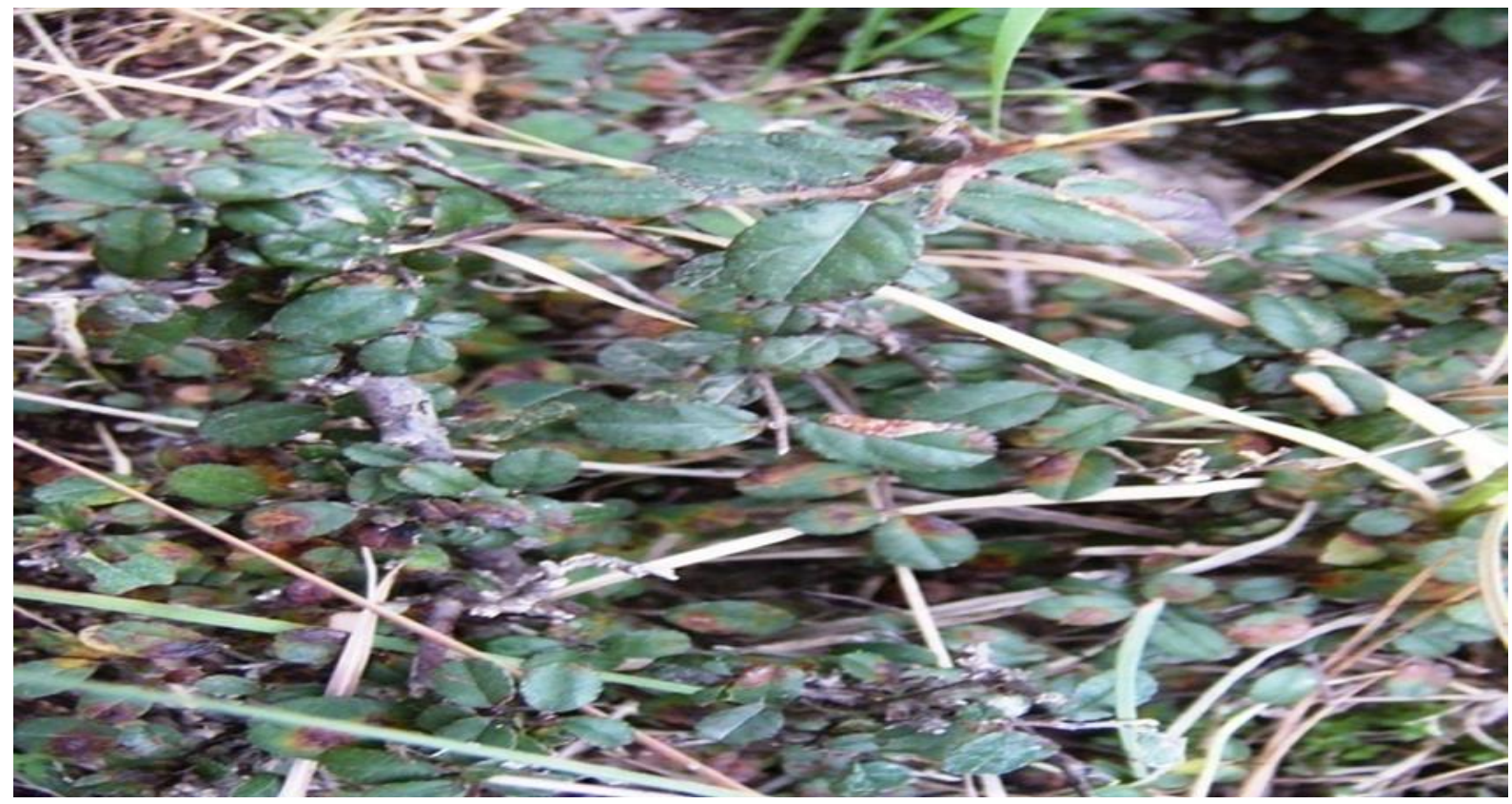

Figure 13. View of heavily grazed Monotheca buaxifolia

Infrequent species

The infrequent species shared 19 species (24.67\%). These include Cymbopogon jwarancusa, Cynodon dactylon, Heteropogon contortus, Achyranthes aspera, Amaranthus caudatus, Launaea procumbens,
Carthamus oxycantha, Chenopodium album, Convolvulus arvensis, Euphorbia helioscopia, Fumaria indica, Ajuga bracteosa, Malva neglecta,Boerhavia procumbens, Oxalis corniculata, Rumex hastatus, Solanum nigrum, Solanum 
surattense and Tribulus terrestris (Table 1). In this dry arid environment, there is always a deficiency of forage and livestock graze every green soft textured plant (Figure 12, 13), resulting in their reduction and hampered regeneration, leading them to the infrequent category. [21] revealed that inhabitants of Kot Manzaray Baba valley use 82 plant species for different purposes. Vegetation of the area was under severe biotic pressure due to deforestation and overgrazing. [22] studied the patterns of species composition and diversity in the lesser Himalayan subtropical forests of Kashmir in relation to environmental variables and underlying anthropogenic influence. Severe grazing intensity was observed at all the study sites.

According to the present studies, various parameters including over-exploitation, extensive harvesting, population explosion, low regeneration, poverty, ignorance, anthropogenic disturbances (Figure 11), deforestation, improper way of collection, fuel wood usage, habitat loss (Figure 7, 9), overgrazing (Figure 3), use as timber, medicinal usage, furniture, land sliding (Figure 9), road expansions (Figure 10) and unwanted incidental fire (Figure 5) are contributing to make the important plants threatened. Moreover, due to unsustainable and unscientific utilization, the forest resources are under immense pressure and are disappearing at an alarming rate.

\section{Conclusions}

It is concluded that flora of district Malakand comprise of diverse plants. The sampled localities revealed that $25 \%$ species were endangered, $29 \%$ vulnerable, $15 \%$ rare while $24 \%$ infrequent. The main causes for plant species vulnerability were recorded as overgrazing and extensive anthropogenic activities.

\section{Authors' contributions}

Designed \& idea of the experiments: Z Muhammad, collected plant materials: H Ali, Identification of the specimen: W
Muhammad, analyzed data: Z Muhammad \& A Majeed, Wrote Paper: Z Muhammad.

\section{References}

1. Lyaruu HV (2010). The influence of soil characteristics on plant species diversify and distribution patterns in Western Serengeti, Tanzania. 5(3): 234-241.

2. Ahmad SS \& Quratulann (2011). Vegetation classification in Ayubia National Park, Pakistan, using ordination methods. Pak J Bot 43(5): 2315-2321.

3. Akeroyd J (2002). A rational look at extinction. Plant Talk 28: 35-37.

4. Akhtar N, Rashid A, Murad W \& Bergmeier E (2013). Diversity and use of ethno-medicinal plants in the region of Swat, North Pakistan. J of Ethnobiology and Ethnomedicine 9.

5. Ali SI \& Qaisar M (1995- 2009). Flora of Pakistan. Pakistan Agricultural Research Council, Islamabad.

6. Bano A, Ayub M, Rashid S, Sultana S \& Sadia H (2013). Ethnobotany and conservation status of floral diversity of Himalayan Range of Azad Jammu and Kashmir, Pakistan. Pak J Bot 45(SI): 243-251.

7. Farooq S, Khan AZ, Yousaf M \& Fazal H (2010). Phytosociological study of Push Ziarat area (Shawal) in the South Waziristan, Pakistan. Pak J Weed Sci Res 16 (1): 47-55.

8. Hamayun M, Khan SA, Sohn EY \& Lee I (2006). Folk medicinal knowledge and conservation status of some economically valued medicinal plants of District Swat, Pakistan. Lyonia 11(2): 101-113.

9. Jan G, Jan FG, Hamayun M, Khan K \& Khan A (2014). Diversity and conservation status of vascular plants of Dir Kohistan valley, Khyber Pakhtunkhwa Province. J of Biodiversity and Environ Sci 5(1): 164-172.

10. Khan (2013). Dimension and composition of plant life in Tehsil Takht- 
e-Nasrati, District Karak, Khyber Pakhtunkhwa, Pakistan.”. PhD Thesis. Department of Botany, University of Peshawar, Peshawar, Khyber Pakhtunkhwa, Pakistan.

11. Khan NA \& Shah M (2013). Ecotaxonomic study of family Brassicaceae of District Mardan, Khyber Pakhtunkhwa, Pakistan. Pak J Life Sci 1(1): 28-35.

12. Khan M \& Hussain F (2013). Conservation status of plant species in Tehsil Takht-e-Nasrati, District Karak, Khyber Pakhtunkhwa, Pakistan. Inter J of Biod and Conser 5(1): 20-26.

13. Khan M \& Musharaf S (2014). Conservation position of plant species in Tehsil Katlang, District Mardan, Pakistan. Medicinal Plant Res 4(7): 5560.

14. Khan M \& Musharaf S (2015). Ethnomedicinal and conservation status of plant species in Tehsil Takht Bhai, District Mardan, Pakistan. Inter Letters of Natural Sci 37: 18-29.

15. Khan M, Hussain F \& Musharaf S (2014). Floristic composition and ecological characteristics of Shahbaz Garhi, District Mardan, Pakistan. Global $J$ of Sci Frontier Res C 14(1): 7-17.

16. Murad W, Ahmad A, Gilani SA \& Khan MA (2011). Indigenous knowledge and folk use of medicinal plants by the tribal communities of Hazar Nao Forest, Malakand District, North Pakistan. $J$ of Medicinal Plants Res 5(7): 1072-1086.

17. Nasir E \& Ali SI (1971-2007). Flora of West Pakistan. Department of Botany, University of Karachi, Karachi, Pakistan.

18. IUCN (2001). IUCN Red List Categories and Criteria: Version 3.1. IUCN Species Survival Commission. IUCN. Gland. Switzerland and Cambridge, UK 2: 30.

19. Nazir A, Malik RN \& Ajaib M (2012). Phytosociological studies of the vegetation of Sarsawa hills District Kotli, Azad Jammu \& Kashmir. Biologia 58(1\&2): 123-133.

20. Shaheen H \&Shinwari ZK (2012). Phytodiversity and endemic richness of Karambar lake vegetation from Chitral, Hindukush-Himalayas. Pak J Bot 44(1): 15-20.

21. Shah M \& Hussain F (2012). Conservation assessment of plant resources of Chakesar valley, District Shangla, Khyber Pakhtunkhwa, Pakistan. Pak J Bot 44(SI): 179-186.

22. Sharma S, Rathi N, Kamal B, Pundir D, Kaur B \& Arya S (2010). Conservation of biodiversity of highly important medicinal plants of India through tissue culture technology- A review. Agric Biol $J N A m$ 1(5): 827-833. 